\title{
Exercise test is essential in LV-only fusion CRT pacing without right ventricle lead
}

This article was published in the following Dove Press journal:

Clinical Interventions in Aging

\author{
Cristina Vacarescu' \\ Dragos Cozma ${ }^{1,2}$ \\ Lucian Petrescu ${ }^{1,2}$ \\ Simona Dragan ${ }^{1,2}$ \\ Cristian Mornos ${ }^{1,2}$ \\ Simina Crisan ${ }^{1,2}$ \\ Horea Feier ${ }^{1,2}$ \\ Mihai-Andrei Lazar' \\ Ramona Alina Cozlac ${ }^{1,2}$ \\ Constantin Tudor Luca ${ }^{1,2}$ \\ 'Cardiology Department, Victor Babeș \\ University of Medicine and Pharmacy, \\ Timișoara, Romania; ${ }^{2}$ Cardiology \\ Department, Institute of Cardiovascular \\ Diseases, Timișoara, Romania
}

Purpose: Left ventricle (LV)-only pacing is non-inferior to biventricular pacing but permanent fusion pacing is needed to ensure cardiac resynchronization therapy (CRT) responsiveness. The role of systematic exercise testing (ET) in these patients has not been established. This study was designed to assess clinical and therapeutic implications (device programming/drugs) of systematic ET in patients requiring fusion-pacing CRT without an right ventricle (RV) lead.

Methods: Consecutive patients with a right atrium/LV-only dual-chamber (DDD) pacing system were included. Prospective data were obtained: device interrogation, ET, and echocardiography at every 6-month follow-up visit. CRT assessment during ET included maximal heart rate, beat-to-beat echocardiography analysis of LV fusion pacing, LV loss of capture, and improvement in exercise capacity. If LV loss of capture or unsatisfactory LV fusion pacing occurred, reprogramming was individualized for each patient and ET redone.

Results: A total of 55 patients ( 29 male) aged $62 \pm 11$ years were included. During follow-up ( $39 \pm 18$ months), a total of 235 ETs were performed, with mean exercise load $6.4 \pm 1.3$ metabolic equivalents of task $(118 \pm 35 \mathrm{~W}$, maximal heart rate $119 \pm 17$ beats/min). Twenty patients $(36 \%)$ had inadequate pacing or loss of LV capture during ET, due to exceeding the maximum tracking rate $(11 \%)$, chronotropic incompetence $(7 \%)$, and LV pacing outside the fusion-pacing band (18\%), caused by physiological shortening of the PR interval or exagerated LV preexcitation during maximum exercise. Post-ET CRT-device optimization included reprogramming of rate-adaptive atrioventricular interval (total decrease $23 \pm 8 \mathrm{~ms}$ ), individualized programming of maximum tracking rate, or rate-response function. Drug optimization was performed in $32 \%$ of patients, and ET redone in $36 \%$.

Conclusion: In one of three ETs, an intervention in device and medication optimization was done to ensure a better outcome. Routine ET should be a standard approach to maximize fusion-pacing CRT response during follow-up.

Keywords: cardiac resynchronization therapy, LV-only pacing, exercise test, constant fusion pacing

\section{Introduction}

Cardiac resynchronization therapy (CRT) using triple-chamber cardiac devices is the current standard treatment in heart-failure guidelines; nevertheless, left ventricle (LV)-only pacing is accepted as noninferior to classic biventricular pacing. ${ }^{1,2}$ Moreover, in a recent review prepared on behalf of the European Heart Rhythm Association Education Committee, Burri et al concluded that LV-only pacing may lead to better CRT outcomes and decrease the number of nonresponders. ${ }^{3}$

Fusion-pacing CRT in sinus rhythm has been studied in patients with biventricular pacemakers, ${ }^{4,5}$ and algorithms like adaptive CRT were designed to promote
Correspondence: Dragos Cozma Cardiovascular Diseases, I3A Gheorghe Adam Street, Timișoara 3003 I0, Romania Tel +40722956370

Fax +40 256207362

Email cdcardiologist@gmail.com 
LV-only pacing in specific conditions. ${ }^{6}$ Using bicameral devices with the right atrium (RA)/LV achieves fusion pacing only in patients with normal atrioventricular (AV) conduction, and is an elegant and safe alternative to classic triple-chamber CRT, with an obvious condition of close to $100 \%$ fusion-pacing stability. ${ }^{7}$ CRT programming immediately after implantation may change during follow-up, and an optimal setting determined at rest may be different during exercise. ${ }^{8}$ How and when we need to optimize CRT is an area of ongoing debate, and even with the advancements achieved thus far, a gold standard is lacking. Optimal exercise CRT programming and tailoring to the individual patient have still not been described. ${ }^{9,10}$

Our assumption is that exercise testing (ET) can be an important follow-up investigation with powerful implications in achieving constant fusion pacing. The aim of this study was to assess and detect CRTprogramming troubleshooting in a population with CRT indication and normal AV conduction in a realworld setting using ET as the principal tool in optimizing RA/LV-only CRT devices.

\section{Methods}

This study was prospectively designed to quantify exercise functional capacity and to detect rhythm changes, such as loss of ventricular capture, sinus tachycardia above upper tracking limit, or shortening/lengthening of the intrinsic PR interval during ET. Patients with CRT-pacemaker indication and normal AV conduction (PR interval $<250 \mathrm{~ms}$ ) on optimal medical treatment for at least 3-6 months without significant coronary artery disease or atrial fibrillation susceptibility (severe biatrial dilatation, longer interatrial conduction time ${ }^{11}$ ) implanted with RA/LV-only DDD pacing systems at Timişoara Institute of Cardiovascular Diseases during 2012-2018 were included. The condition to perform ET was at least 1 month of stable medical state without changes in medication or hospitalization due to heart-failure (HF) decompensation. Exclusion criteria were inability to perform ET due to noncardiac physical limitation (eg, orthopedic pathology) or decompensated HF.

At each follow-up, patients were checked for LV threshold, spontaneous AV interval, capture quality, adequate response of pacemaker functioning to exercise, percentage of pacing, electrocardiography (ECG) 12lead pacing on/off, and review of pharmacological therapy. For all patients included in the study, at every device interrogation we certified that both atrial sensing and atrial pacing provided the same pattern of fusion pacing on surface ECG. Complete transthoracic echocardiography using standard views and techniques was also performed in all patients, with special focus during follow-up on LV systolic function parameters: LV endsystolic volume, LV end-diastolic volume, and LV ejection fraction.

Cycle-ergometry ET was performed on a GE exercise system with an increase in workload of $25 \mathrm{~W}$ for each 3-minute exercise stage. Blood pressure and 12-lead ECG were continuously recorded. Exercise capacity was measured in metabolic equivalents of task at peak exercise. CRT assessment during ET included maximal heart rate, beat-to-beat ECG analysis of true LV-fusion pacing, loss of LV capture, and improvement in exercise capacity. Even if ET at baseline offered a clear advantage regarding PR interval behavior during ET, most patients were not able to exercise before implantation, and thus isolated ET sessions could not be analyzed.

In patients with loss of LV capture during exercise by exceeding maximum tracking rate (MTR), drug optimization was performed (uptitration of $\beta$-blocker [BB]/ adding ivabradine) associated with increasing MTR limit by 15 beats/min. When chronotropic incompetence was noted, rate-response function was programmed and ET redone immediately.

All ET sessions were performed with live monitoring of the PM, and one of the main objectives was to maintain constant fusion pacing, both at rest and during maximal exercise. We defined the fusion-pacing band according to existing evidence ${ }^{12}$ as the range of $\mathrm{AV}$ intervals at which surface ECG (mainly in lead $\mathrm{V}_{1}$ ) has an intermediate morphology between native left bundle-branch block (upper limit of the band) and fully paced right BBB (lower limit). Two situations were noted outside the fusion pacing band: in patients with loss of capture by physiological shortening of PR interval or improper fusion pacing (rest-to-exercise variations regarding R-wave reduction in $\mathrm{V}_{1}$ ), the dynamic AV interval was progressively decreased by $20 \mathrm{~ms}$, and in patients with important LV preexcitation during maximum exercise (right BBB appearance in $V_{1}$ ), the dynamic AV interval was progressively increased by $20 \mathrm{~ms}$.

The AV interval was programmed individually and beat-to-beat ECG analysis performed to ensure adequate and constant fusion pacing. The stability of the spontaneous $\mathrm{PR}$ interval was obtained by $\mathrm{BB} \pm$ ivabradine-dose titration, such that in shorter PR-interval patients the dose 
of $\mathrm{BB}$ alone was increased, while in longer PR-interval patients ivabradine without $\mathrm{BB}$ or in combination with lower doses of $\mathrm{BB}$ was given. Various BBs have been used; however, for study purposes carvedilol-equivalent doses were calculated using the algorithm described by Cohen-Solal et al. ${ }^{13}$

In patients where only device optimization was needed, ET was redone immediately after reprogramming to ensure adequate fusion pacing. In patients with drug-therapy changes, ET was redone at an interval no longer than 2 weeks. The study was conducted in accordance with the 1964 Declaration of Helsinki, and the protocol was approved by the ethical review committee of Timişoara Institute of Cardiovascular Diseases (1622/26.03.2014). Written informed consent was obtained from all patients included in the study.

\section{Statistical analysis}

Data are expressed as means \pm SD for continuous variables and proportions for categorical variables. Continuous variables were compared between groups using unpaired $t$-tests (variables with normal distribution) or Mann-Whitney $U$ tests (abnormally distributed variables). Proportions were compared using $\chi^{2}$ and Fisher's exact test. $P<0.05$ was considered significant. All analyses were carried out with the SPSS version 18.0 (SPSS Inc., chicago, IL, USA).

\section{Results}

A total of 55 patients with RA/LV DDD pacing systems were initially included. Seven patients had been lost by the end of the study (in five patients, nonsudden cardiac death occurred, and two patients developed orthopedic pathology). Basic demographic and echocardiography data are presented in Table 1. All patients were receiving optimal medical therapy, including HF medication (BB/ivabradine, ACE inhibitors/ARBs, antialdosteronics) individually titrated according to clinical and paraclinical variables (heart rate, blood pressure, renal function). During follow-up, we noted clinical and paraclinical improvement, and medication was uptitrated to target doses where possible; however, the main goal of BB and ivabradine titration was to obtain stable $\mathrm{AV}$ conduction to ensure constant fusion pacing both at rest and during exercise.

The following Pacing systems were implanted: Biotronik (eight patient), Medtronic (eight patients), St Jude Medical (34 patients), Boston Scientific (two patients), and Sorin Group (three patients). Bipolar RA/LV leads were used. No short- or long-term device-related complications were noted (eg, phrenic nerve stimulation, pocket infections). At baseline, all
Table I Baseline demographic data

\begin{tabular}{|c|c|c|}
\hline & & $\begin{array}{l}\text { All patients } \\
(n=55)\end{array}$ \\
\hline \multicolumn{2}{|l|}{ Mean age (years) } & $62 \pm 11$ \\
\hline \multicolumn{2}{|l|}{ Male, n (\%) } & $30(55 \%)$ \\
\hline \multirow{2}{*}{$\begin{array}{l}\text { NYHA functional } \\
\text { class } \\
(n, \%)\end{array}$} & II & $24(44 \%)$ \\
\hline & III & $31(56 \%)$ \\
\hline \multirow[t]{2}{*}{ Electrocardiography } & $\begin{array}{l}\text { PR interval }(\mathrm{ms}) \text {, mean } \pm \\
\mathrm{SD}\end{array}$ & $186 \pm 32$ \\
\hline & $\begin{array}{l}\text { QRS complex (ms), mean } \\
\pm \mathrm{SD}\end{array}$ & $163 \pm 17$ \\
\hline \multirow{3}{*}{$\begin{array}{l}\text { Mitral regurgitation } \\
(n, \%)\end{array}$} & Mild & $6(11 \%)$ \\
\hline & Moderate & $27(49 \%)$ \\
\hline & Severe & $22(40 \%)$ \\
\hline \multicolumn{2}{|l|}{ Hypertension, n (\%) } & $20(36 \%)$ \\
\hline \multicolumn{2}{|c|}{ Diabetes mellitus, $n$ (\%) } & $20(36 \%)$ \\
\hline \multicolumn{2}{|c|}{ Chronic kidney disease, n (\%)* } & $23(42 \%)$ \\
\hline \multirow[t]{6}{*}{ Echographic data } & $\operatorname{LVEDD}(\mathrm{cm})$, mean \pm SD & $6.4 \pm 0.9$ \\
\hline & $\operatorname{LVEF}(\%),{ }^{* *}$ mean \pm SD & $27 \pm 5.2$ \\
\hline & $\operatorname{LVEDV}(\mathrm{mL})$, mean $\pm \mathrm{SD}$ & $243.2 \pm 82$ \\
\hline & LVESV $(\mathrm{mL})$, mean \pm SD & $182.4 \pm 73$ \\
\hline & LAV $(m L)$, mean $\pm S D$ & $104.9 \pm 34$ \\
\hline & $\mathrm{sPAP}(\mathrm{mmHg})$, mean $\pm \mathrm{SD}$ & $46.6 \pm 15$ \\
\hline
\end{tabular}

Notes: *Defined as reduction in creatinine clearance $<90 \mathrm{~mL} / \mathrm{min}$. None of the patients in our cohort had creatinine clearance $<30 \mathrm{~mL} / \mathrm{min}$. **Measured by modified Simpson's method.

Abbreviations: LAV, left atrial volume; LVEDD, left ventricular end-diastolic diameter; LVEDV, left ventricular end-diastolic volume; LVEF, left ventricular ejection fraction; LVESV, left ventricular end-systolic volume; NYHA, New York Heart Association; sPAP, systolic pulmonary artery pressure.

devices were programmed at a rest rate of 60 beats $/ \mathrm{min}$ and MTR of 130 beats/min. Individualized AV intervals with AV pace of $147 \pm 22 \mathrm{~ms}$ and sensed $\mathrm{AV}$ of $119 \pm 25 \mathrm{~ms}$ (dynamic AV interval at baseline equal to sensed $\mathrm{AV}$ ), allowing fusion pacing in all patients.

During follow-up of $39 \pm 18$ months, 235 ET sessions were done, with a mean of $118 \pm 35 \mathrm{~W}(6.4 \pm 1.3$ metabolic equivalents of task) and a peak heart rate of $119 \pm 17$ beats/ min. Inadequate pacing or loss of LV capture was noted in 20 patients (36\%), with causes identified being MTR exceeded (11\% of patients), chronotropic incompetence $(7 \%$ of patients; Figure 1), LV pacing outside the fusion-pacing band (18\% of patients), including loss of capture by 
A

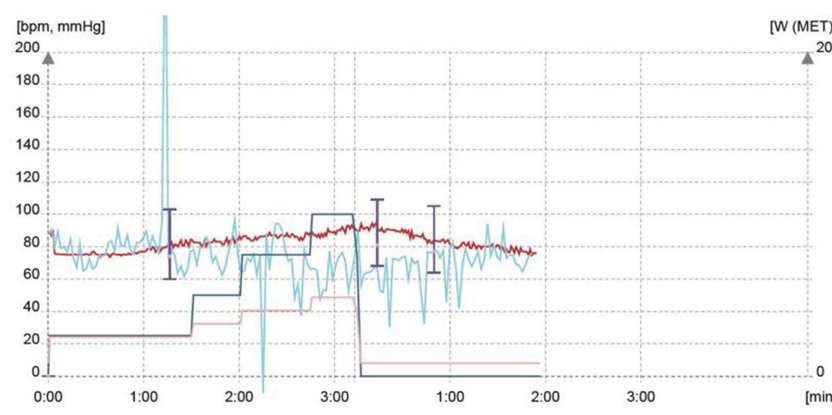

B

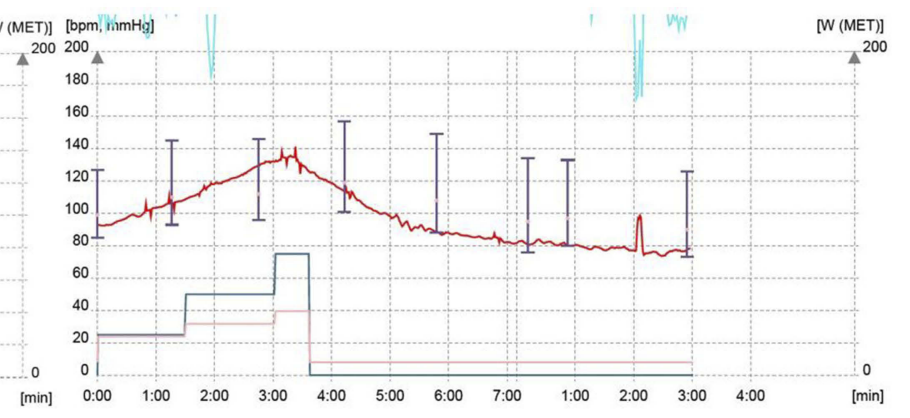

Figure I Heart-rate diagram during exercise (red line).

Notes: (A) Example of a patient with chronotropic incompetence; (B) heart-rate acceleration after reprogramming rate-response function.

physiological shortening of PR interval or improper fusion pacing (rest-to-exercise variations in $\mathrm{R}$-wave reduction in $\mathrm{V}_{1}, 15 \%$ of patients) and important $\mathrm{LV}$ preexcitation during maximum exercise with right $\mathrm{BBB}$ appearance in $\mathrm{V}_{1}(3 \%$ of patients; Figure 2). Post-ET CRT optimization was performed by increasing MTR at 145 beats/min and reprogramming dynamic AV interval. After ET optimization, we registered a decrease in dynamic AV interval of $23 \pm 8 \mathrm{~ms}$.

Medication changes included BB uptitration with addition/uptitration of ivabradine in six patients (11\%), due to LV-capture loss by exceeding MTR, and uptitration of $\mathrm{BB}$ in another nine patients $(16 \%)$, due to loss of
LV capture by physiological shortening of PR interval or improper fusion pacing. In three patients $(5 \%)$ treated with $\mathrm{BB} / \mathrm{ivabradine}$, chronotropic incompetence was noted and ivabradine excluded from treatment. Comparative data representing baseline and follow-up demographic characteristic, detailed changes in medication, and device reprogramming after performing ET are presented in Table 2.

During ET, episodes of paroxysmal atrial fibrillation at maximum exercise were noted in two patients $(4 \%)$, and amiodarone was introduced without recurrence at further examinations. In one patient (2\%), after 3 years' follow-up

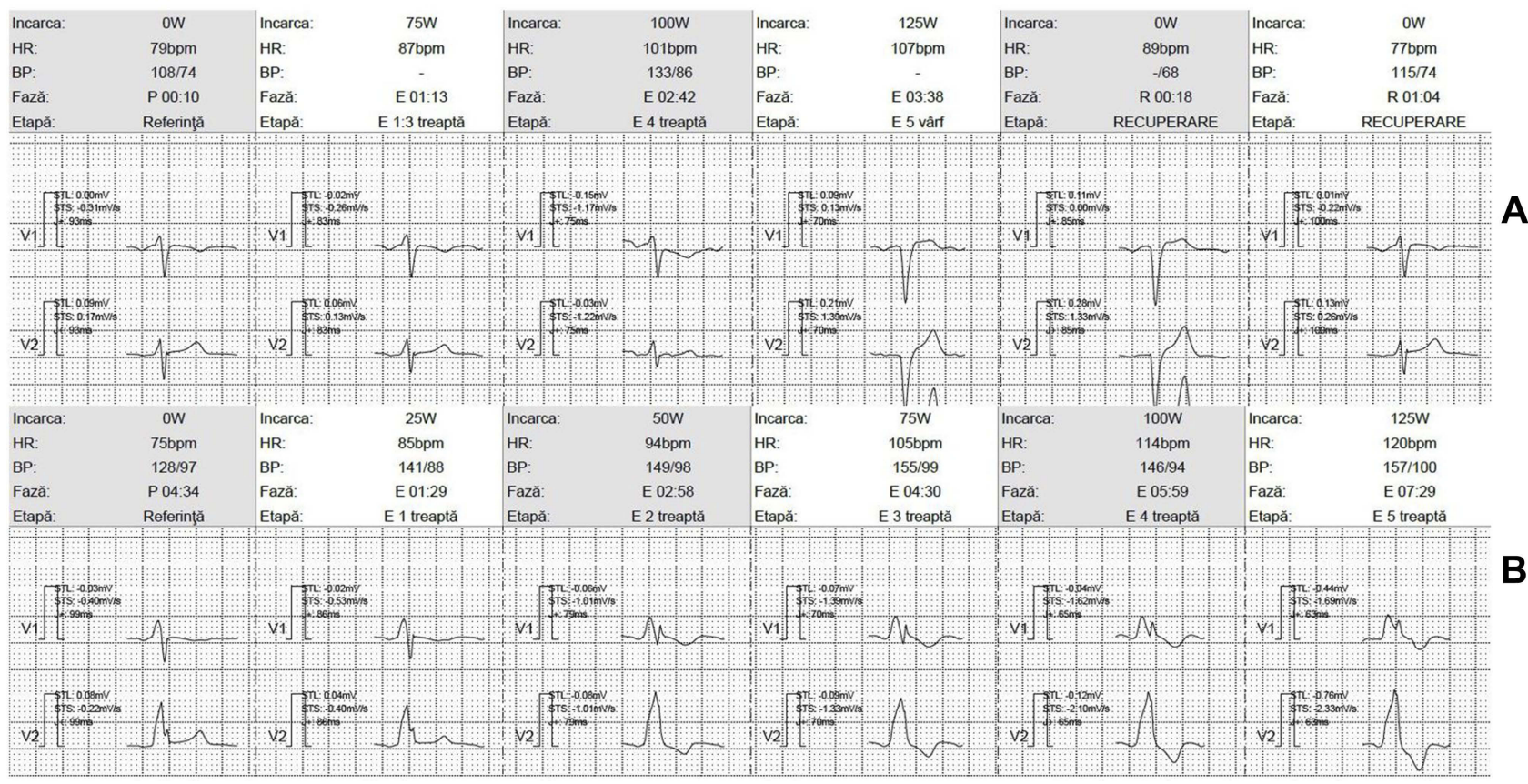

Figure 2 LV pacing during exercise outside the fusion pacing band.

Notes: $(\mathbf{A})$ Rest-to exercise-variations regarding $R$-wave reduction in $V_{1} ;(B)$ important $L V$ preexcitation during maximum exercise $\left(R B B B\right.$ appearance in $\left.V_{1}\right)$. Incarca $=$ exercise load; HR=heart rate; BP=blood pressure; Fază=phase; Etapă=stage; treaptă=level; RECUPERARE=recovery; vârf=peak; Referință=reference; bpm=beats per minute. Abbreviations: LV, left ventricle; RBBB, right bundle-branch block. 
Table 2 Comparative data representing baseline and follow-up demographic characteristics, detailed changes in medication, and device reprogramming after ET $(n=55)$

\begin{tabular}{|c|c|c|c|c|}
\hline & Baseline & $\begin{array}{l}\text { Post-ET optimization } \\
\text { (follow-up } 39 \pm \text { I } 8 \text { months) }\end{array}$ & Relative change, \%* & $P$-value \\
\hline LVEF, mean \pm SD & $27 \pm 5.2$ & $37 \pm 7.9$ & $27 \%$ & $<0.0001$ \\
\hline LVEDV $(\mathrm{mL})$, mean \pm SD & $243.2 \pm 82$ & $|93.7 \pm 8|$ & $20 \%$ & 0.0028 \\
\hline LVESV $(\mathrm{mL})$, mean $\pm S D$ & $182.4 \pm 73$ & $113 \pm 63$ & $38 \%$ & $<0.0001$ \\
\hline LVESV reduction $\geq 15 \%$, patients & - & 16 & $29 \%$ & - \\
\hline Increasing MTR, patients & - & 6 & $11 \%$ & - \\
\hline Programming rate-response function, patients & - & 4 & $7 \%$ & - \\
\hline Reprogramming dynamic $A V$ interval, patients & - & 10 & $18 \%$ & - \\
\hline Dynamic AV interval, ms & $119 \pm 25$ & $96 \pm 17$ & $19 \%$ & $<0.0001$ \\
\hline BB therapy, patients (\%) & $45(82 \%)$ & $54(98 \%)$ & $16 \%$ & - \\
\hline Maximal target-dose BB, patients (\%) & $6(11 \%)$ & $21(38 \%)$ & $27 \%$ & - \\
\hline Carvedilol-equivalent dose, $\mathrm{mg}$ (mean $\pm \mathrm{SD}$ ) & $6.7 \pm 7$ & $17.5 \pm 12$ & $62 \%$ & $<0.0001$ \\
\hline Ivabradine therapy, patients (\%) & $24(44 \%)$ & $27(49 \%)$ & $5 \%$ & - \\
\hline Ivabradine daily dose, $\mathrm{mg}$, mean $\pm \mathrm{SD}$ & $4.6 \pm 2$ & $5.3 \pm 2$ & $13 \%$ & 0.0692 \\
\hline
\end{tabular}

Abbreviations: $A V$, atrioventricular; $B B, \beta$-blocker; ET, exercise testing; LAV, left atrial volume; LVEF, left ventricular ejection fraction; LVEDV, left ventricular end-diastolic volume; LVESV, left ventricular end-systolic volume; MTR, maximum tracking rate.

we noted at ET alternating fusion narrow QRS and wide complete LV-capture QRS. Pacemaker inhibition during exercise revealed Mobitz II second-degree AV block (Figure 3). After amiodarone and BB cessation, the AV block persisted and the patient upgraded to a classic triplechamber CRT device, without complications.

At the end of follow-up, $36 \%$ of patients needed CRTdevice reprogramming, while in $32 \%$ of patients medication optimization was performed. We also found statistical significance for several echocardiography parameters: LV end-systolic volume ( $182.4 \pm 73$ vs $113 \pm 63, P<0.0001)$, LV end-diastolic volume (243.2 \pm 82 vs $193.7 \pm 81, P<0.0028)$, and LV ejection fraction $(27 \pm 5.2$ vs $37 \pm 7.9, P<0.0001)$. The main outcome of this systematic follow-up was a $90 \%$ responder rate in our cohort.

\section{Discussion}

This study shows that ET represents an important tool for follow-up and optimization in CRT devices that provide LV-only fusion pacing. The usefulness of ET in CRT follow-up was highlighted in patients with classic triple-chamber CRT devices and biventricular pacing, and even in small series, a significant percentage of patients need device reprogramming after ET. ${ }^{14,15}$ With the ultimate goal of maximizing CRT benefits, the current guideline recommends empirical programming of 100-120 ms sensed AV delay and simultaneous biventricular stimulation; however, the population of $30 \%$ nonresponders remains the Achilles heel of CRT, with no clear evidence of optimization or improvement. ${ }^{2}$
A

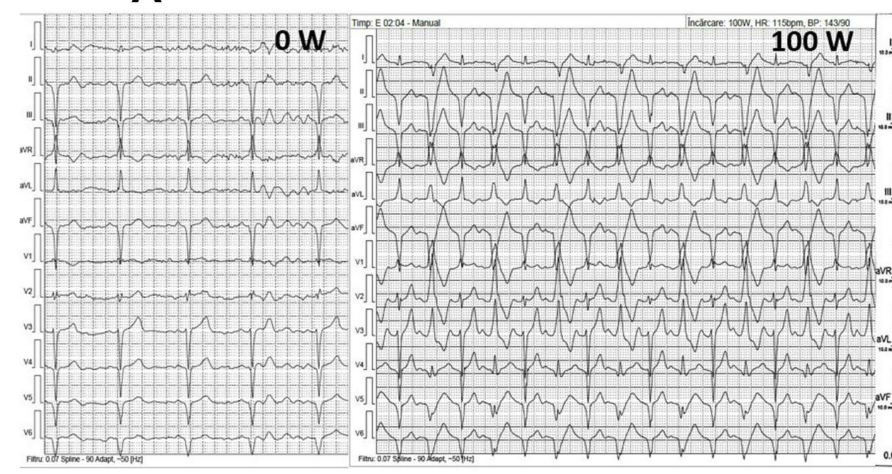

C

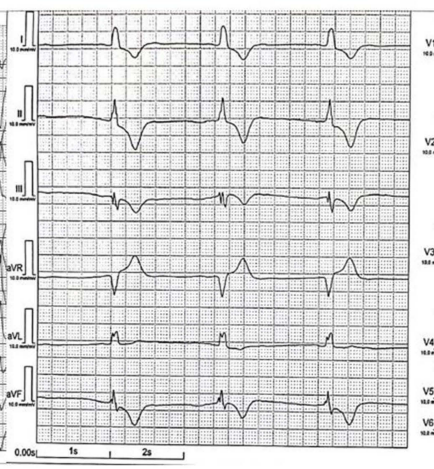

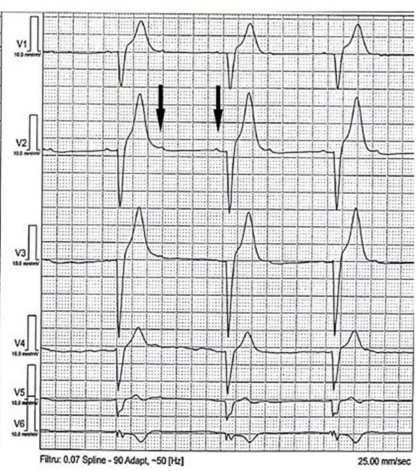

Figure 3 Mobitz II second degree AV block occurrence during ET.

Notes: (A) LV-only fusion pacing at the beginning of ET; (B) at $100 \mathrm{~W}$, fusion narrow QRS complexes and wide complete LV capture QRS complexes; (C) pacemaker inhibition with Mobitz II second-degree AV block.

Abbreviations: AV, atrioventricular; ET, exercise testing; LV, left ventricle. 
Leclercq identifies the most common reasons for loss of capture at exercise in biventricular devices: loss of atrial sensing, frequent premature ventricular complexes, atrial tachyarrhythmia, and spontaneous AV conduction that is more rapid than the programmed AV delay. ${ }^{16}$ All these issues come up in RA/LV-only CRT devices, with the addition of a specific problem: maintaining constant fusion pacing both at rest and during exercise. Physiological shortening of the AV interval with increasing heart rate has been well documented in normal subjects, but also in patients with New York Heart Association III/IV HF on $\beta$-antagonist or antiarrhythmic therapy, is mostly observed in earlier stages of exercise, and represents an established component of dual-chamber pacing for bradycardia as rate-adaptive or dynamic $\mathrm{AV}$ intervals. ${ }^{17,18}$

Although fusion pacing has clear evidence of substantially improving the structural responder rate, ${ }^{19-21}$ the variability of $\mathrm{AV}$ conduction still remains the main concern regarding LV-only fusion pacing, both in the short and long term, due to changes in disease state, exercise capacity, and medication. ${ }^{3}$ The algorithm described by the investigators of an adaptive-CRT trial, ${ }^{6}$ which promotes intrinsic conduction and reduces RV pacing, has been proven save and effective for heart rate $<100 / \mathrm{min}$ (empirical limit based on a 22-patient hemodynamic study), and the only algorithm designed for DDD LV-only patients without an RV lead was described in 2007.,22 Developing a device-based algorithm for beat-by-beat monitoring of effective LV pacing is feasible ${ }^{23}$ and still a matter of high interest; nevertheless, with a lack of large controlled randomized trials and guideline-based evidence, ET remains an important real-life investigation that can assess constant fusion pacing.

A mainstay class of drug therapy in HF, $\beta$-blockers have important implications in providing fusion-pacing stability by their effect of slowing AV conduction. Moreover, a combination of early coadministration of BB-ivabradine for patients with acute HF with reduced ejection fraction showed 1-year improvement in LV systolic function. ${ }^{24}$ Nevertheless, recent data have highlighted that uptitration of HF medication is still a major drawback in real-world practice, and several studies have shown a general effort toward optimization of therapy after CRT with important benefits in reducing HF hospitalization and increasing survival, with a special focus on BB uptitration as an independent predictor of improved HF prognosis. ${ }^{25-28}$ Although such medications as ACE inhibitors/ARBs, loop diuretics, and antialdosteronics are of utmost importance in HF patients and changes pertaining to these drugs were also performed in our cohort during follow-up according to functional status and clinical/paraclinical variables, this study aimed to assess the importance of $\mathrm{BB} / \mathrm{ivabradine}$ therapy and CRT-device reprogramming after systematic ET in maintaining constant CRT fusion pacing, which has proven to be a real need in clinical practice.

The small, selective cohort and lack of randomization are important limitations; however, the study highlights the importance of finding better methods of evaluation and optimization of LV-only CRT fusion pacing. Although the end point of the study was not a clinical one in terms of HF hospitalizations or mortality, we succeeded in demonstrating that ET has powerful implications regarding device and medication optimization in fusion-pacing CRT patients. We found at the end of the study an important statistical significance regarding echocardiography parameters of LV systolic function, correlating with a $90 \%$ responder rate in our cohort; we can only assume this finding is based on a careful follow-up to permanently reassess treatment and device optimization.

\section{Conclusion}

A third of CRT fusion-pacing patients need device and medication optimization after ET. Routine ET has important implications in maintaining constant fusion pacing, and should be a standard approach to maximize CRT response during follow-up.

\section{Acknowledgment}

This research received no external funding.

\section{Author contributions}

All authors contributed toward data analysis, drafting and revising the paper, gave final approval of the version to be published and agree to be accountable for all aspects of the work.

\section{Disclosure}

The authors report no conflicts of interest in this work.

\section{References}

1. Ponikowski P, Voors AA, Anker SD, et al. ESC Scientific Document Group; ESC Guidelines for the diagnosis and treatment of acute and chronic heart failure: the task force for the diagnosis and treatment of acute and chronic heart failure of the European Society of Cardiology. Eur Heart J. 2016;37(27):2129-2200. doi:10.1093/eurheartj/ehw128

2. Brignole M, Auricchio A, Baron-Esquivias G, et al. ESC guidelines on cardiac pacing and cardiac resynchronization therapy: the task force on cardiac pacing and resynchronization therapy of the European Society of Cardiology (ESC). Eur Heart J. 2013;34(29):2281-2329.

3. Burri H, Prinzen FW, Gasparini M, et al. Left univentricular pacing for cardiac resynchronization therapy. Europace. 2017;19(6):912-919. 
4. Gasparini M, Bocchiardo M, Lunati M, et al. Comparison of 1-year effects of left ventricular and biventricular pacing in patients with heart failure who have ventricular arrhythmias and left bundle-branch block: the $\mathrm{Bi}$ vs Left Ventricular Pacing: an International Pilot Evaluation on Heart Failure Patiens with Ventricular Arrhythmias (BELIEVE) multicenter prospective randomized pilot study. Am Heart J. 2006;152(1):155.e1-155.e7.

5. Boriani G, Kraning W, Donal E, et al. A randomized double blind comparison of biventricular versus left ventricular stimulation for cardiac resynchronization therapy: the Biventricular versus Left Univentricular Pacing with ICD back-up in Heart Failure Patients (BLEFT HF) trial. Am Heart J. 2010;159(6):1052-1058.e1.

6. Martin DO, Lemke B, Birnie D, et al. Investigation of a novel algorithm for synchronized left-ventricular pacing and ambulatory optimization of cardiac resynchronization therapy: results of the adaptive CRT trial. Heart Rhythm. 2012;9(11):1807-1814.

7. Cozma D, Vacarescu C, Petrescu L, et al. CRT pacing: midterm follow-up in LV only pacing without RV lead in patients with normal AV conduction. J Clin Med. 2018;7(12):531.

8. Bertini M, Delgado V, Bax JJ, et al. Why, how and when do we need to optimize the setting of cardiac resynchronization therapy? Europace. 2009;11:v46-v57.

9. Chousou PA, Pugh PJ. How to deliver optimal cardiac resynchronisation therapy. Heart. 2018;104:1300-1307.

10. Grimaldi A, Gorodeski EZ, Rickard J. Optimizing cardiac resynchronization therapy: an update on new insights and advancements. Curr Heart Fail Rep. 2018;15(3):156-160. doi:10.1007/s11897-018-0391-y

11. Cozma D, Kalifa J, Pescariu S, et al. Can simple doppler measurements estimate interatrial conduction time? Pacing Clin Electrophysiol. 2003;26(1P2):436-439.

12. Gianfranchi L, Bettiol K, Sassone B, et al. Fusion beat in patients with heart failure treated with left ventricular pacing: may ECG morphology relate to mechanical synchrony? A pilot study. Cardiovasc Ultrasound. 2008;6:1. doi:10.1186/1476-7120-6-1

13. Cohen-Solal A, Jacobson FA, Piña LI. Beta blocker dose and markers of sympathetic activation in heart failure patients: interrelationships and prognostic significance. ESC Heart Fail. 2017;4(4):499-506. doi:10.1002/ehf2.12153

14. de Riva-Silva M, López Gil M, Fontenla-Cerezuela A, et al. Usefulness of exercise test in cardiac resynchronization therapy follow-up. Rev Esp Cardiol. 2013;66:912-913. doi:10.1016/j.rec.2013.05.021

15. Valzania C, Eriksson MJ, Boriani G, et al. Cardiac resynchronization therapy during rest and exercise: comparison of two optimization methods. Europace. 2008;10(10):1161-1169. doi:10.1093/europace/ eun216
16. Leclercq C. Problems and troubleshooting in regular follow-up of patients with cardiac resynchronization therapy. Europace. 2009;11: v66-v71. doi:10.1093/europace/eup124

17. Lee JU, Kim KS, Kim JH, et al. PR interval behavior during exercise stress test. Korean J Intern Med. 1995;10(2):137-142.

18. Luceri RM, Brownstein SL, Vardeman L, et al. PR interval behavior during exercise: implications for physiological pacemakers. Pacing Clin Electrophysiol. 1990;13(12 Pt 2):1719-1723.

19. Vatasescu R, Berruezo A, Mont L, et al. Midterm 'super-response' to cardiac resynchronization therapy by biventricular pacing with fusion: insights from electro-anatomical mapping. Europace. 2009;11(12):1675-1682. doi:10.1093/europace/eup333

20. Guo T, Li R, Zhang L, et al. Biventricular pacing with ventricular fusion by intrinsic activation in cardiac resynchronization therapy. Int Heart J. 2015;56(3):293-297.

21. Thibault B, Ducharme A, Harel F, et al. Left ventricular versus simultaneous biventricular pacing in patients with heart failure and a QRS complex $\geq 120$ milliseconds. Circulation. 2011;124 (25):2874-2881.

22. Biffi M, Bertini M, Ziacchi M, et al. Left ventricular pacing by automatic capture verification. Europace. 2007;9:1177-1781.

23. Ghosh S, Stadler RW, Mittal S. Automated detection of effective left-ventricular pacing: going beyond percentage pacing counters. Europace. 2015;17(10):1555-1562.

24. Hidalgo FJ, Carrasco F, Castillo JC, et al. Early Therapy with Beta Blockers Plus Ivabradine Versus Beta Blockers Alone in Patients Hospitalised with Heart Failure and Reduced Ejection Fraction (ETHIC-AHF Study): results at one-year follow-up. Int $\mathrm{J}$ Clin Cardiol. 2017;93:093.

25. Crişan S, Petrescu L, Lazăr MA, et al. Reduced ejection fraction heart failure-new data from multicenter studies and national registries regarding general and elderly populations: hopes and disappointments. Clin Interv Aging. 2018;13:651-656.

26. Massoullié G, Chouki C, Mulliez A, et al. Effect of optimization of medical treatment on long-term survival of patients with heart failure after implantable cardioverter defibrillator and cardiac resynchronization device implantation (from the french national EGB database). Am J Cardiol. 2018;121(6):725-730.

27. Witt CT, Kronborg MB, Nohr EA, et al. Optimization of heart failure medication after cardiac resynchronization therapy and the impact on long-term survival. Eur Heart J Cardiovasc Pharmacother. 2015;1 (3):182-188

28. Nabeta $\mathrm{T}$, Inomata $\mathrm{T}$, Iida $\mathrm{Y}$, et al. Prognostic significance of beta-blocker up-titration in conjunction with cardiac resynchronization therapy in heart failure management. Heart Vessels. 2016;31 (7):1109-1116
Clinical Interventions in Aging

\section{Publish your work in this journal}

Clinical Interventions in Aging is an international, peer-reviewed journal focusing on evidence-based reports on the value or lack thereof of treatments intended to prevent or delay the onset of maladaptive correlates of aging in human beings. This journal is indexed on PubMed Central, MedLine, CAS, Scopus and the Elsevier
Bibliographic databases. The manuscript management system is completely online and includes a very quick and fair peer-review system, which is all easy to use. Visit http://www.dovepress.com/ testimonials.php to read real quotes from published authors. 